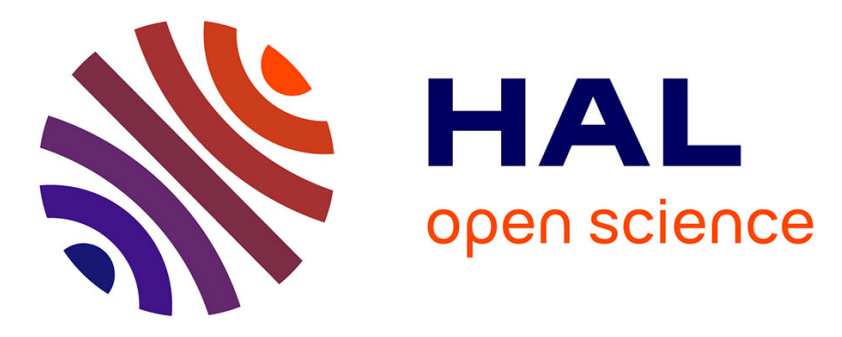

\title{
Hidden magnetic frustration by quantum relaxation in anisotropic Nd-langasite
}

Virginie Simonet, Rafik Ballou, Julien Robert, Benjamin Canals, Françoise

Hippert, Pierre Bordet, Pascal Lejay, Peter Fouquet, Jacques Ollivier, Daniel

Braithwaite

\section{To cite this version:}

Virginie Simonet, Rafik Ballou, Julien Robert, Benjamin Canals, Françoise Hippert, et al.. Hidden magnetic frustration by quantum relaxation in anisotropic Nd-langasite. Physical Review Letters, 2008, 100, pp.237204. 10.1103/PhysRevLett.100.237204 . hal-00266071v2

\section{HAL Id: hal-00266071 \\ https://hal.science/hal-00266071v2}

Submitted on 13 Jun 2008

HAL is a multi-disciplinary open access archive for the deposit and dissemination of scientific research documents, whether they are published or not. The documents may come from teaching and research institutions in France or abroad, or from public or private research centers.
L'archive ouverte pluridisciplinaire HAL, est destinée au dépôt et à la diffusion de documents scientifiques de niveau recherche, publiés ou non, émanant des établissements d'enseignement et de recherche français ou étrangers, des laboratoires publics ou privés. 


\title{
Hidden magnetic frustration by quantum relaxation in anisotropic Nd-langasite
}

\author{
V. Simonet, ${ }^{1}$ R. Ballou, ${ }^{1}$ J. Robert,${ }^{1}$ B. Canals,${ }^{1}$ F. Hippert,${ }^{2}$ P. \\ Bordet, ${ }^{1}$ P. Lejay, ${ }^{1}$ P. Fouquet, ${ }^{3}$ J. Ollivier, ${ }^{3}$ and D. Braithwaite ${ }^{4}$ \\ ${ }^{1}$ Institut Néel, CNRS/UJF, B.P. 166, 38 042 Grenoble Cedex 9, France \\ ${ }^{2}$ LMGP (CNRS), Grenoble Institute of Technology, 38016 Grenoble, France \\ ${ }^{3}$ Institut Laue-Langevin, BP 156, 38042 Grenoble Cedex 9, France \\ ${ }^{4}$ CEA-Grenoble, INAC/SPSMS/IMAPEC, 17 rue des Martyrs, 38054 Grenoble cedex, France
}

(Dated: June 13, 2008)

\begin{abstract}
The static and dynamic magnetic properties of the $\mathrm{Nd}_{3} \mathrm{Ga}_{5} \mathrm{SiO}_{14}$ compound, which appears as the first materialization of a rare-earth kagome-type lattice, were re-examined, owing to contradictory results in the previous studies. Neutron scattering, magnetization and specific heat measurements were performed and analyzed, in particular by fully taking account of the crystal electric field effects on the $\mathrm{Nd}^{3+}$ ions. One of the novel findings is that the peculiar temperature independent spin dynamics observed below $10 \mathrm{~K}$ expresses single-ion quantum processes. This would short-circuit the frustration induced cooperative dynamics, which would emerge only at very low temperature.
\end{abstract}

PACS numbers: 75.10.Dg,75.40.Gb,61.05.fg

The kagome antiferromagnet is the prototype of geometrically frustrated systems in 2 dimensions. It is predicted that its ground state, in the classical case, remains disordered and is highly degenerate [1]. The investigation of such a spin-liquid state is still impeded by the few available experimental materializations. Various secondary perturbations actually lead, in most known compounds, to long-range order (LRO) or spin glass phase. This occurs, though, at a temperature well below the onset of magnetic correlations and is often accompanied by a dynamical behavior persisting below the transition temperature. These dynamics are characterized by a slowing down of the magnetic fluctuations which rounds off onto a relaxation plateau [3]. Although rather generic, the origin of this peculiar relaxation is still debated.

In all the kagome compounds so far studied the magnetic entities that form the frustrated lattice of cornersharing triangles are $3 \mathrm{~d}$ transition metal ions with weak magneto-crystalline anisotropy. Inversely, in the 3 dimensional compounds of the pyrochlore family, if rareearth ions with strong magneto-crystalline anisotropy occupy a frustrated lattice of corner-sharing tetrahedra. This leads to a wider variety of magnetic behaviors. Most striking, for instance, was the discovery of the spin ice state in $\mathrm{Ho}_{2} \mathrm{Ti}_{2} \mathrm{O}_{7}$, where the frustration arises from the combined effects of multi-axial anisotropy and ferromagnetic interactions [- It was expected, in contrast, that the multi-axial anisotropy would release the geometric frustration of antiferromagnetic interactions in $\mathrm{Tb}_{2} \mathrm{Ti}_{2} \mathrm{O}_{7}$, but this compound exhibits no LRO and, instead, shows intriguing spin dynamics well below the Curie-Weiss (CW) temperature [5, 6. It was recently argued that this compound would form a quantum spin ice from dynamical frustration induced through crystal field (CF) excitations and quantum many-body effects [7].

The lately discovered $\mathrm{Nd}_{3} \mathrm{Ga}_{5} \mathrm{SiO}_{14}$ (NGS) compound, which belongs to the langasite family, provides with the unique opportunity to study the role of a strong magnetocrystalline anisotropy in a geometrically frustrated 2 dimensional lattice. In this compound the rare-earth $\mathrm{Nd}^{3+}$ ions fully occupy a lattice showing the kagome cornersharing triangles connectivity if only first neighbor interactions are taken into consideration [8].

The magnetic properties of NGS powder samples and single crystal were investigated in the $1.6-400 \mathrm{~K}$ temperature range [8]. Neutron diffraction and magnetization measurements on the powders confirmed that NGS does not order in a Néel phase nor shows any signature of a spin glass behaviour. Magnetization measurements on the single-crystal (cf. Fig. 乐) revealed a strong magnetocrystalline anisotropy associated with CF effects on the ground multiplet $\mathrm{J}=9 / 2$ of the $\mathrm{Nd}^{3+}$ ions. A mean field quantitative analysis of the magnetic susceptibility at high temperature taking into account only the quadrupolar contribution to the $\mathrm{CF}$ yielded a $\mathrm{CW}$ temperature $\theta=-52 \mathrm{~K}$, leading to anticipate rather strong antiferromagnetic interactions between the Nd moments. These were described as coplanar rotators in the kagome planes. A change in the anisotropy occurs around $33 \mathrm{~K}$, below which the c-axis (perpendicular to the kagome planes) becomes the macroscopic magnetization axis.

Recently, a neutron scattering investigation of NGS at very low temperature was reported [9]. A weak ring of diffuse magnetic scattering was observed at $46 \mathrm{mK}$, whose momentum-transfer $(Q)$ dependence was found in agreement with calculated magnetic scattering maps from spin-liquid models [10]. It was inferred from additional neutron spin-echo (NSE) experiments, that the associated magnetic state would be highly dynamical. The authors also claimed to have evidenced a magnetic field induced phase transition featured by a plateau of reduced magnetization, approximately half the moment value of the free $\mathrm{Nd}^{3+}$ ion, in agreement with magnetization measurements $\left(1.6 \mu_{B}\right.$ at $\left.1.6 \mathrm{~K}\right)[8]$. Another recent investiga- 


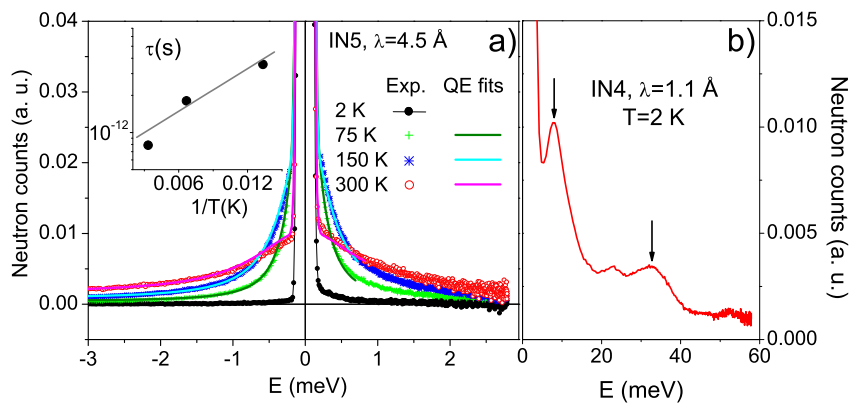

FIG. 1: (Color online) Time-of-flight spectra, $Q$-integrated in the range $[0.34,2.55] \AA^{-1}$. a) Quasielastic magnetic scattering fitted from 75 to $300 \mathrm{~K}$ by a Lorentz function multiplied by the detailed balance factor. The fluctuation time obtained from the inverse HWHM is shown in the inset together with an Arrhenius fit $\left(\tau=\tau_{0} \exp \left(\Delta E / k_{B} T\right)\right.$ with $\Delta \mathrm{E}=133 \mathrm{~K}$ and $\left.\tau_{0}=6.710^{-13} \mathrm{~s}\right)$. b) Two non-dispersive crystal-field level transitions pointed out by the arrows. Note that the feature at $\approx 23 \mathrm{meV}$ was attributed to phonons from the $Q$-dependence.

tion by $\mu \mathrm{SR}$ and NMR spectroscopy [11] established that the magnetic fluctuations slow down as the temperature is decreased to reach a relaxation plateau below $10 \mathrm{~K}$, interpreted as a signature of magnetic frustration in this cooperative system. Some usual signatures of frustration therefore appeared to have been observed in NGS, magnetic spatial correlations and a fluctuating ground state, but inconsistencies soon emerge from close inspection. In reference 9, no magnetic signal is reported at $46 \mathrm{mK}$ outside an energy window corresponding to fluctuating times larger than $510^{-11} \mathrm{~s}$ but NSE experiments at the same temperature appears to suggest a relaxation faster than $410^{-12} \mathrm{~s}$. In addition, this fast relaxation suggested by the NSE experiment is incompatible with the characteristic fluctuation time of the $\mu$ SR-probed relaxation plateau, $\tau \approx 2.510^{-10} \mathrm{~s}$. This motivated our experimental re-investigation of the spin dynamics in NGS, presented below, as well as a full characterization of the single-ion anisotropy through a complete CF analysis.

Powders of Nd-langasite, synthesized as in ref. 8, were used for magnetization and specific heat measurements and neutron scattering experiments. The spin dynamics were investigated via time-of-flight (ToF) and NSE spectroscopy. The ToF experiments were performed at ILL on the IN4 spectrometer at a wavelength of $1.1 \AA$ and on the IN5 spectrometer with wavelengths between 8.5 and $2.25 \AA$. The ToF spectra reveal a quasielastic (QE) signal, displayed in Fig. 1, whose magnetic origin was inferred from its $Q$-dependence (slight decrease following the $\mathrm{Nd}^{3+}$ squared form factor). The half width at half maximum (HWHM) of the $Q$-integrated spectra decreases from $300 \mathrm{~K}$ to $75 \mathrm{~K}$. An Arrhenius law can be forced to account for the temperature dependence of the relaxation time $\tau=\hbar / H W H M$. This yields an energy barrier of the order of $130 \mathrm{~K}$ for a thermally activated process. At $2 \mathrm{~K}$, no QE signal can be detected any more within the instrumental resolution energy window.

In order to extend the time window for the observations of the dynamics, a NSE experiment was performed on the IN11C spectrometer at ILL using a wavelength of $5.5 \AA$. XYZ polarization analysis was performed to experimentally select the magnetic part of the signal. The instrumental resolution was corrected with the purely elastic magnetic signal of a reference compound. The accessible time window of the NSE technique overlaps the ToF range for short times and the $\mu \mathrm{SR}$ range for long times. The normalized intermediate functions $S(Q, t) / S(Q, 0)$, measured between 1.4 and $100 \mathrm{~K}$, can be fitted by stretched exponential line-shapes $\exp \left(-(t / \tau)^{\beta}\right)$ (cf. Fig. 2). A $\beta$ exponent $\neq 1$ denotes a non-unique relaxation time. It increases gradually from a constant value of 0.5 between 1.4 and $10 \mathrm{~K}$ to 0.8 at $40 \mathrm{~K}$, indicating a change in the dynamics. The small temporal range with non-zero signal at higher temperatures prevented from obtaining a reliable value of $\beta$ which was therefore fixed equal to 0.8 between 60 and $100 \mathrm{~K}$. Above $40 \mathrm{~K}$, the thermal variation of the relaxation time agrees with a thermally activated process $(\ln (\tau) \propto 1 / T)$. We get an energy barrier very close to the one extracted from the ToF QE signal (cf. Fig. 2] [12]. Below $10 \mathrm{~K}$, the relaxation time does no more vary with temperature, revealing a relaxation plateau with a characteristic time $\tau \approx 310^{-10} \mathrm{~s}$, in good agreement with the $\mu \mathrm{SR}$ results [11] (and at variance with the NSE data of ref. 9). An important finding of our NSE experiment is that the magnetic relaxation does not show any detectable $Q$-dependence in the range $\left[0.3 \AA^{-1}, 1.7 \AA^{-1}\right]$, pointing out the absence of spatial correlation down to $1.4 \mathrm{~K}$ since the frequency width is inversely proportional to the $Q$-dependent susceptibility in correlated paramagnet [13. The spatial correlations observed at $46 \mathrm{mK}$ [9] thus would occur in a temperature range much below the onset of the relaxation plateau.

Additional insights about the magnetism of NGS were gained from the ToF scattering at higher energy transfer, specific heat measurements and investigations of magnetically diluted samples. Two CF level transitions can be identified, through their $Q$ dependence in the ToF spectra, around $95 \mathrm{~K}$ (also reported in ref. 9) and $380 \mathrm{~K}$ (cf. Fig. 1). They however are very broad in energy, with a width at least one order of magnitude larger than the instrumental resolution. Quantitative estimations of CF effects suggest that this may be ascribed to the distribution of the charge environments associated with the $\mathrm{Si}^{4+} / \mathrm{Ga}^{3+}$ mixed site [8], which also is responsible for the NMR line broadening reported in ref. 11. The specific heat measurements were performed down to $400 \mathrm{mK}$ in a Quantum Design PPMS. The magnetic contribution was determined after subtraction of the specific heat measured on the isostructural non-magnetic $\mathrm{La}_{3} \mathrm{Ga}_{5} \mathrm{SiO}_{14}$ compound. It exhibits a broad bump ranging from $\approx 100 \mathrm{~K}$ to above $300 \mathrm{~K}$. A strong rise is noticeable below 


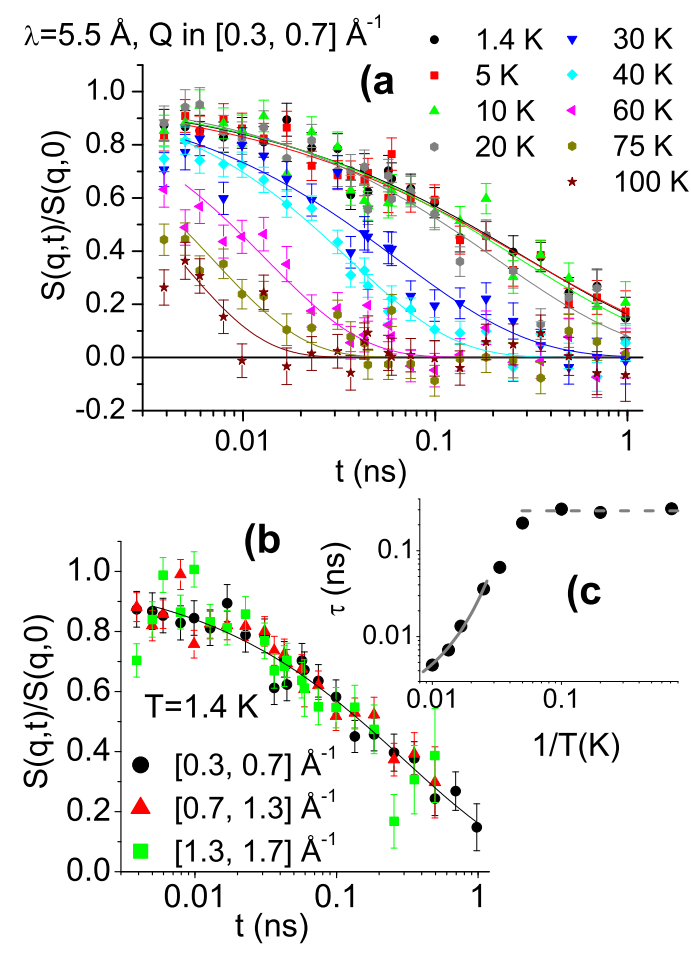

FIG. 2: (Color online) Neutron spin-echo normalized intermediate functions fitted with stretched exponentials (solid lines) for several temperatures (a), and for several $Q$ ranges at 1.4 $\mathrm{K}$ (b). The variation of the relaxation time over the inverse temperature is displayed with an Arrhenius fit (solid line) at high temperatures $\left(\Delta \mathrm{E}=135 \mathrm{~K}, \tau_{0}=1.210^{-12} \mathrm{~s}\right)$ and a dashed line underlying the relaxation plateau below $10 \mathrm{~K}$ (c).

$2 \mathrm{~K}$ as the temperature is decreased down to the lowest values (cf. Fig. 3). We shall discuss this below. Also meaningful were the magnetization measurements on the magnetically diluted $\left(\mathrm{Nd}_{0.01} \mathrm{La}_{0.99}\right)_{3} \mathrm{Ga}_{5} \mathrm{SiO}_{14}$ compound, which were performed in order to isolate the influence of the magneto-crystalline anisotropy. We found out, to our surprise, that its magnetic susceptibility does experimentally not differ from that of NGS (cf. Fig. 3), demonstrating that the exchange interaction between the $\mathrm{Nd}^{3+}$ moments in NGS is certainly much smaller than initially estimated [8], at least not greater than a few $\mathrm{K}$.

The above results were confronted to calculations of the CF effects. In NGS, there are 3 distinct $\mathrm{Nd}^{3+}$ ions per unit cell, each lying on a different 2 -fold axis, interrelated through the 3 -fold $\vec{c}$ axis of the crystal [8], thus giving rise to multi-axial low symmetry $\mathrm{CF}$ potentials. The associated Hamiltonian for each $\mathrm{Nd}^{3+}$ ion in the ground multiplet $J=9 / 2$ can be expressed as $H^{C F}=$ $\sum_{p=1}^{3} \sum_{q=-p}^{p} A_{2 p}^{2 q} O_{2 p}^{2 q}$ with $A_{2 p}^{-2 q}=\left(A_{2 p}^{2 q}\right)^{\star}$, in terms of $O_{n}^{m}$ Racah operators and with respect to a local frame where the 2-fold axis is the quantization axis for the angular momentum [14]. Ignoring exchange interactions this provides with an energy spectrum which can be compared

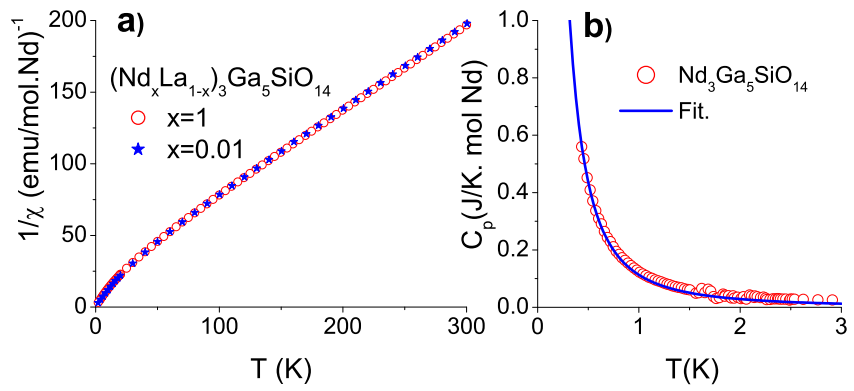

FIG. 3: (Color online) a) Inverse susceptibility of NGS and of a magnetically diluted sample. b) NGS low temperature specific heat fitted by $\mathrm{R}\left(\frac{\Delta \mathrm{E}}{\mathrm{k}_{\mathrm{B}} \mathrm{T}}\right)^{2} \frac{\exp \left(-\frac{\Delta \mathrm{E}}{\mathrm{k}_{\mathrm{B}} \mathrm{T}}\right)}{\left(1+\exp \left(-\frac{\Delta \mathrm{E}}{\mathrm{k}_{\mathrm{B}} \mathrm{T}}\right)^{2}\right.}$ for a Schottky anomaly produced by a two-level system with $\mathrm{R}$ the gas constant and $\Delta \mathrm{E}=235 \mathrm{mK}$.

with the transitions detected in the ToF spectra and can be used to calculate the specific heat. The magnetization and susceptibility are obtained from the eigenfunctions and must be averaged over the $3 \mathrm{Nd}^{3+}$ ions. This forced us to work in a common global frame with the $\vec{c}$ axis as the quantization axis and to perform appropriate rotations of the Racah operators. It was illusory to try to determine the 9 independent $A_{2 p}^{2 q}$ coefficients at once. Thus, in a first step the $A_{2 p}^{0} / A_{2 p}^{2 q}$ ratios were calculated in the point charge model from the first coordination shell of eight $\mathrm{O}^{2-}$ (cf. Fig. A). Qualitatively good results were already obtained, reproducing in particular the crossing of the susceptibility for applied fields parallel and perpendicular to $\vec{c}$. This crossing is due to the $4^{\text {th }}$ order terms of $H^{C F}$. The $A_{2 p}^{2 q}$ coefficients were, in a second step, slightly varied in order to better account for all the experimental constrains (details of the calculations will be given elsewhere). With the best set of $A_{2 p}^{2 q}$ parameters, the energy spectrum consists of 5 degenerate doublets, the first two being separated from the ground state doublet by $90 \mathrm{~K}$ and $324 \mathrm{~K}$ respectively, in rather good agreement with the ToF spectra considering the width of the measured excitations. The ground state in the global frame with the $\vec{c}$ quantization axis is a combination of $\mid \pm 7 / 2>$, $\mid \pm 5 / 2>$ and $\mid \pm 3 / 2>$ states with a maximum weight for the $\mid \pm 5 / 2>$ state. The good agreement between the calculated and measured magnetic isotherms and susceptibility is shown in Fig. 14. A first observation is that the low temperature reduced moment must be attributed to a CF effect, the so-called field-induced magnetic phase [9, 11] being hence a polarized paramagnet, at least at $1.6 \mathrm{~K}$, as in $\mathrm{Tb}_{2} \mathrm{Ti}_{2} \mathrm{O}_{7}$ 16. A second observation is that the susceptibility reaches the high temperature behaviour above $2000 \mathrm{~K}$. This regime occurs thus at a temperature much higher than the one associated to the highest crystal field level energy $(665 \mathrm{~K})$. This is a consequence of the large $6^{\text {th }}$ order terms, shifting the inverse susceptibil- 


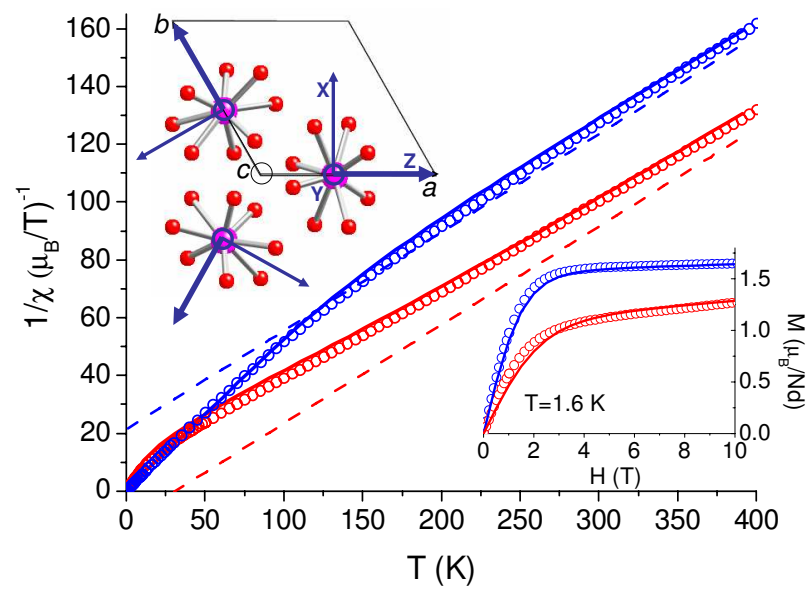

FIG. 4: (Color online) Full CF calculation (solid lines) of the inverse susceptibility (main frame) and magnetic isotherms (inset) with the magnetic field parallel (red) or perpendicular (blue) to the kagome planes compared to single-crystal measurements (circles) [8]. The dashed lines are high temperature expansion calculations. The three $\mathrm{Nd}^{3+}$ are shown in their local frame with their first coordination $\mathrm{O}^{2-}$ shells.

ities upward, which explains why the previous estimation of the exchange interactions went erroneous despite the almost linear variations of the inverse susceptibility [8].

The CF analysis confirms the smallness of the exchange interaction in consistency with the absence of spatial correlations down to $1.4 \mathrm{~K}$. This suggests a single-ion origin for the measured magnetic relaxation both in the varying and independent temperature regimes, as probed by the neutron spectroscopy. The first excited doublet of the calculated CF spectrum lies at an energy in qualitative agreement with the barrier extracted from the ToF and NSE measurements at temperatures above $40 \mathrm{~K}$. The thermally activated dynamics in this temperature range are most probably mediated by the spin-phonon interaction [17]. On decreasing the temperature, this progressively slows down, but below $10 \mathrm{~K}$ is short-circuited by another process with a temperature independent relaxation time. This strongly suggests quantum relaxation phenomena 18, 19 and calls to mind the dynamical cross-over observed in spin ice compounds 20]. The origin of this relaxation is still unclear but could be related to the only feature not accounted for by the CF calculation: the rise of the specific heat at very low temperature. A splitting of the ground state doublet was suggested in ref. 9 from extrapolation of Tof data to zero-field under magnetic field. Assuming this suggestion, we fitted the specific heat by a $T^{-2}$ law in agreement with the tail of a Schottky anomaly associated with a two-level system (cf. Fig. 3). This gives an energy gap of $\approx 235 \mathrm{mK}$, in good agreement with the reported zero-field extrapolated splitting [9]. Although the cause of this splitting is unidentified, we know that it should necessarily break the time reversal symmetry, since the $\mathrm{Nd}^{3+}$ are Kramers ions. An interaction involving odd powers of the ladder operators of the $\mathrm{Nd}^{3+}$ angular momentum would do, by providing with non-diagonal matrix elements mixing the two orthogonal states of the doublet. The consequences of this low temperature dynamics on the cooperative magnetic phase, expected when the exchange interactions and the geometric frustration become significant, can now be addressed. A cooperative dynamical behaviour presenting longer fluctuation times than the fluctuations created by the proposed single-ion quantum process would indeed be hindered. This, thus, queries the nature of the phase associated to the ring-like $Q$-distribution at $46 \mathrm{mK}$ [9].

In conclusion, the experimental re-investigations of the kagome-like rare-earth NGS compound combined with $\mathrm{CF}$ analysis have allowed featuring its magnetism on firm grounds, solving previous inconsistencies. The interpretation of the relaxation plateau of the moment dynamics in terms of cooperative processes is ruled out. Its origin must rather be sought from single-ion quantum processes. The effects of the geometrical frustration then would be hidden emerging only at very low temperature.

This work was financially supported by the ANR 06BLAN-01871.

[1] D. A. Huse et al., Phys. Rev. B 45, R7536 (1992); J. T. Chalker et al., Phys. Rev. Lett. 68, 855 (1992).

[2] J. Villain, J. Phys. (Paris) 41, 1263 (1980).

[3] H. Mutka et al., Phys. Rev. Lett. 97, 047203 (2006).

[4] M. J. Harris, et al., Phys. Rev. Lett. 79, 2554 (1997); A. P. Ramirez, Nature 399, 333 (1999).

[5] J. S. Gardner et al., Phys. Rev. Lett. 82, 1012 (1999).

[6] M. J. P. Gingras et al., Phys. Rev. B 62, 6496 (2000).

[7] H. R. Molavian et al., Phys. Rev. Lett. 98, 157204 (2007).

[8] P. Bordet et al., J. Phys.: Condens. Matter 18, 5147 (2006).

[9] H. D. Zhou et al., Phys. Rev. Lett. 99, 236401 (2007).

[10] J. Robert et al., Phys. Rev. Lett. 96, 197205 (2006); Phys. Rev. Lett. 97, 259901(E) (2006).

[11] A. Zorko et al., Phys. Rev. Lett. 100, 147201 (2008).

[12] The factor 2 in the $\tau_{0}$ values obtained from the Tof and NSE data may be due to the distribution of relaxation times. This is revealed from the fits of the NSE data by stretched exponentials whose Fourier Transforms are not rigorously the Lorentz functions used in the Tof analysis.

[13] S. W. Lovesey, Theory of Neutron Scattering from Condensed Matter, vol. 2, Oxford Science Publications.

[14] D. J. Newman and B. Ng, Crystal Field Handbook, Cambridge University Press (2000).

[15] $A_{2}^{0}=-4.7, A_{2}^{2}=-3.076-3.565 \mathrm{i}, A_{4}^{0}=0.22, A_{4}^{2}=0.318+0.019 \mathrm{i}$, $A_{4}^{4}=0.119+0.096 \mathrm{i}, \quad A_{6}^{0}=0.028, \quad A_{6}^{2}=0.023+0.043 \mathrm{i}$, $A_{6}^{4}=0.014-0.005 \mathrm{i}, A_{6}^{6}=0.012+0.019 \mathrm{i}$

[16] K. C. Rule et al., Phys. Rev. Lett. 96, 177201 (2006).

[17] J. Villain et al., Europhys. Lett. 27, 159 (1994).

[18] L. Thomas et al., Nature 383, 145 (1996).

[19] R. Giraud et al., Phys. Rev. Lett. 87, 057203 (2001).

[20] G. Ehlers et al., J. Phys.: Condens. Matter 15, L9 (2003). 